\title{
Computational Screening of Metal Organic Frameworks for Mixed Matrix Membrane
}

\section{Applications}

\author{
Ilknur Erucar† and Seda Keskint* \\ $\dagger$ Department of Computational Sciences and Engineering, \\ Koç University, 34450, Istanbul, Turkey \\ †Department of Chemical and Biological Engineering, \\ Koç University, 34450, Istanbul, Turkey
}

Submitted to Journal of Membrane Science

\begin{abstract}
In this study, detailed molecular simulations were used to examine the challenge of selecting metal organic frameworks (MOFs) as filler particles in high performance mixed matrix membranes (MMMs) for separation of $\mathrm{H}_{2}$ from $\mathrm{CH}_{4}$. The predictions of theoretical permeation models for gas permeability were compared with the experimental data of IRMOF-1/Matrimid, CuBTC/PSF, CuBTC/PDMS and Cu-BPY-HFS/Matrimid MMMs. The good agreement between experiments and our theoretical predictions motivated us to estimate performances of one hundred and nineteen new MOF-based MMMs, composed of seventeen different MOFs and seven different polymers. We identified several MOF-based MMMs exhibiting very high $\mathrm{H}_{2}$ selectivity and $\mathrm{H}_{2}$ permeability relative to pure polymer membranes. Finally, we discussed the ways of selecting appropriate MOFs as filler particles for a given polymer matrix which will result in MMMs with promising properties for $\mathrm{H}_{2}$ separations.
\end{abstract}

Keywords: metal organic framework; membrane; polymer; molecular simulation

\footnotetext{
${ }^{*}$ Corresponding author. Email: skeskin@ku.edu.tr Phone:+902123381362 Fax:+902123381548
} 


\section{TOC Graphic}

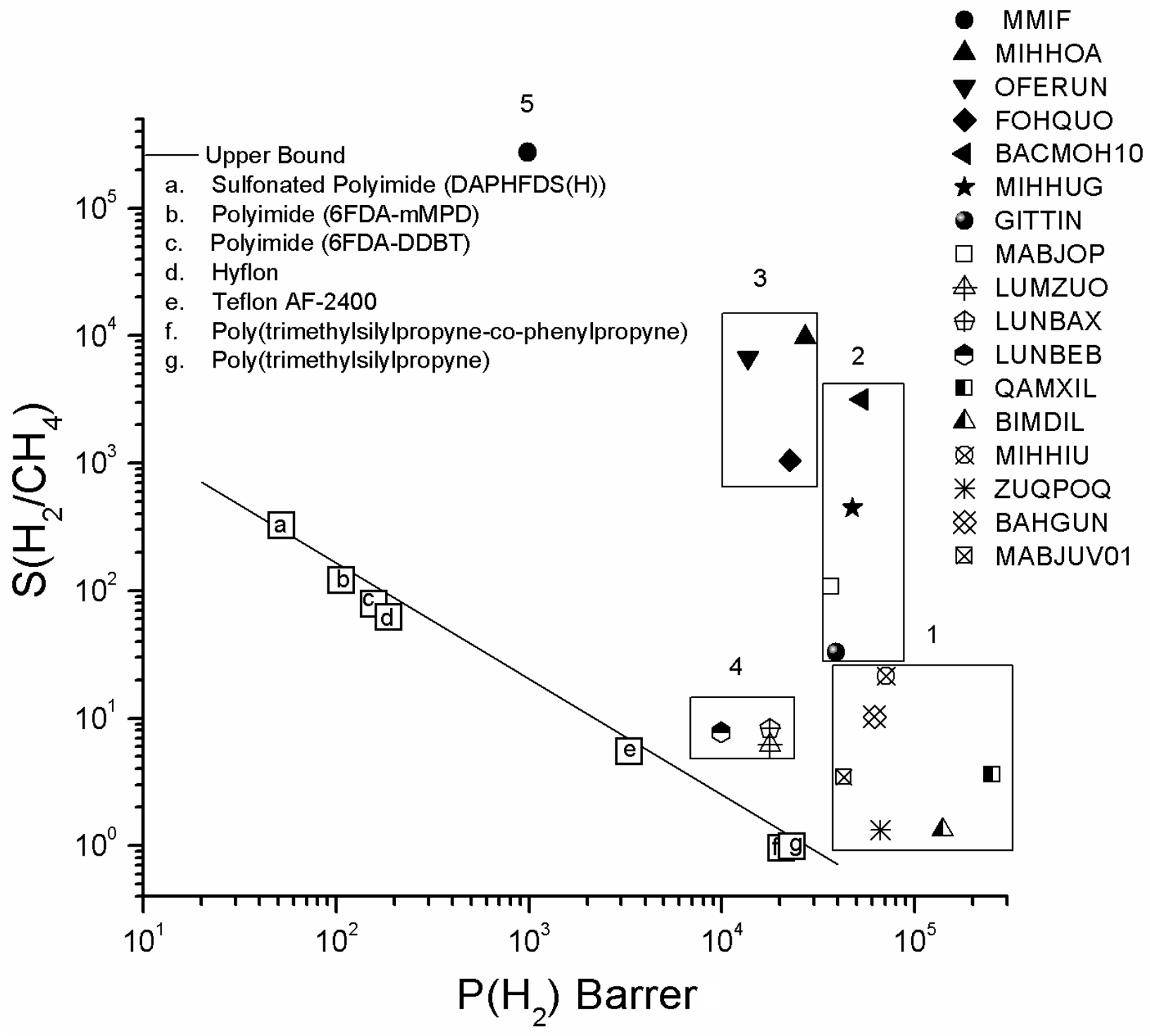




\section{Introduction:}

There is an increasing demand for hydrogen in various fields including fuel cells, semiconductor processing, petrochemical industry since hydrogen is regarded as a renewable and ecologically clean energy source. Hydrogen is widely produced by steam reforming of methane. In order to achieve high purity hydrogen production, it is crucial to separate hydrogen from methane. Polymeric membranes have been commercially used for separation of $\mathrm{H}_{2}$ from $\mathrm{CH}_{4}$ because these membranes offer ease of fabrication and low cost. However, polymeric membranes have a trade-off between selectivity and permeability, the key parameters for gas separations. Polymeric membranes offering high selectivity exhibit low permeability which results in large surface areas and high capital cost. Robeson used large collections of experimental data to demonstrate the inverse relationship between selectivity and permeability of polymeric membranes [1,2]. Freeman presented a theory showing that the upper bound described by Robeson is a consequence of the strong size sieving nature of the stiff chain glassy polymer materials [3,4]. Development of membrane materials that can exceed Robeson's upper bound to achieve $\mathrm{H}_{2} / \mathrm{CH}_{4}$ separations with high $\mathrm{H}_{2}$ selectivity and high $\mathrm{H}_{2}$ permeability has been one of the main targets of membrane research.

One way of overcoming the selectivity/permeability trade-off is to make membranes from non-polymeric materials such as zeolites [5], carbon nanotubes [6,7]. Unfortunately, scale up difficulties and high manufacturing costs present fundamental challenges in the commercial implementation of these materials as membranes [8]. The other way to overcome this tradeoff is to combine polymers with non-polymeric materials to make mixed matrix membranes (MMMs). MMMs are heterogeneous membranes in which organic or inorganic fillers are embedded into a polymer matrix. These membranes show great promise for short-term commercial implementation because fabrication of MMMs on large scales can readily be envisioned with relatively minor adaptation of existing commercial technology $[9,10]$. Many different types of non-polymeric materials such as non-porous silica particles [11], carbon molecular sieves [12,13], zeolites [14,15,16,17], fullerenes [18] and carbon nanotubes [19] have been used as filler particles in MMMs. Metal organic frameworks (MOFs) are new alternatives to be used as filler particles in MMMs. MOFs are recognized with their great potential in gas storage and gas separation applications due to their well defined pores and large surface areas [20]. MOFs are composed of metal ligand complexes forming vertices of a framework that is connected with organic linkers. The main advantage of MOFs over well known nanoporous materials is the ability to tune their physical and chemical properties 
during synthesis by changing the combination of metals and organic linkers. Rational combination of different metal ions and organic linkers in the synthesis of MOFs results in materials with various pore size and connectivity, offering theoretically infinite number of possible structures [21].

Many recent studies showed that it is feasible to use MOFs as filler particles in MMM applications to enhance the performance of pure polymers for achieving high gas selectivity and high gas permeability. Car et al. [22] prepared MMMs using CuBTC as filler particles in polysulfone (PSF) and in polydimethylsiloxane (PDMS). They reported that both $\mathrm{H}_{2}$ permeability and $\mathrm{H}_{2} / \mathrm{CH}_{4}$ selectivity of MMMs are higher than those of pure polymers. Hu et al. [23] synthesized $\mathrm{CuBTC} /$ polyimide $\mathrm{MMMs}$ and showed that $\mathrm{H}_{2}$ permeance and selectivity are increased compared to pure polyimide. Zhang et al. [24] incorporated Cu-BPY-HFS into Matrimid to study the pure gas permeation of $\mathrm{H}_{2}, \mathrm{~N}_{2}, \mathrm{O}_{2}, \mathrm{CH}_{4}$, and $\mathrm{CO}_{2}$ as well as the separation of binary gas mixtures. They concluded that $\mathrm{H}_{2}$ permeability is increased whereas a slight decrease is observed for $\mathrm{H}_{2} / \mathrm{CH}_{4}$ selectivity. Basu et al. [25] synthesized CuBTC/Matrimid and CuBTC/Matrimid/PSF membranes and observed increases in selectivity and permeance of $\mathrm{CO}_{2}$ compared to the reference polymer membranes. Perez and co-workers [26] used the most widely studied MOF, IRMOF-1, as filler particles in Matrimid to study the same gas pairs mentioned above and reported increases in $\mathrm{H}_{2}$ permeability and $\mathrm{H}_{2} / \mathrm{CH}_{4}$ selectivity. Adams et al. [27] incorporated CuTPA into polyvinyl acetate and observed that both gas permeability and selectivity were enhanced compared to pure polymer. Yang et al. [28] synthesized zeolite imidazolate framework-7 (ZIF-7)/polybenzimidazole (PBI) composite membranes which showed enhanced $\mathrm{H}_{2}$ permeability and enhanced $\mathrm{H}_{2} / \mathrm{CO}_{2}$ selectivity surpassing PBI membranes. Diaz et al. [29] used PFG NMR techniques to study transport of $\mathrm{CO}_{2}$ in a MMM including ZIF-8 particles. Liu and coworkers [30] incorporated ZIF-8 nanoparticles into a polymethylphenylsiloxane matrix to provide preferential pathways for the permeation of organic compounds whereas Zhang et al. [31] fabricated ZIF-8/6FDADAM polyimide membranes and showed that both propylene selectivity and permeability increase as the amount of ZIF-8 increases in the MMM. Bae and coworkers [32] used ZIF-90 as filler particles in Ultem, Matrimid and 6FDA-DAM polyimide and reported that ZIF90/6FDA-DAM membranes exhibit very high performance for $\mathrm{CO}_{2} / \mathrm{CH}_{4}$ separations. Basu and coworkers [33,34] used CuBTC, MIL-47, MIL-53(Al), ZIF-8 as filler particles in PDMS and Matrimid. 
These attempts of fabricating MOF-based MMMs indicate that it is possible to enhance pure polymers' performance by incorporating MOFs as filler particles into the polymers, however in most cases the increase in $\mathrm{H}_{2}$ permeability is obtained at the expense of a decrease in $\mathrm{H}_{2}$ selectivity. For example, IRMOF-1 and CuBTC are known to exhibit very high gas permeability due to their large pore sizes. These two MOFs do not show a high $\mathrm{H}_{2}$ selectivity over $\mathrm{CH}_{4}$ since both gas species can permeate through the large pores as evidenced by experimental and theoretical studies [35,36]. Selecting the appropriate MOF which can increase both $\mathrm{H}_{2}$ selectivity and $\mathrm{H}_{2}$ permeability compared to the pure polymer for specific separations of interest is crucial but not easy due to the very large number of existing MOFs. There are hundreds of MOFs that could potentially be used as filler particles even if only one polymer is considered. A key challenge in developing new MOF-based MMMs is the need to efficiently screen a variety of MOFs to identify the most promising ones prior to experimental efforts. Therefore, theoretical models that can make quantitative predictions for the performance of new MOF-based MMMs play an important role. Keskin and Sholl [37] introduced a computational method that combines detailed atomic simulations with Maxwell permeation model to provide the first quantitative information for MOF-based MMMs and validated their predictions by comparing with the experimental data of IRMOF-1/Matrimid MMMs for $\mathrm{CO}_{2} / \mathrm{CH}_{4}$ separations. Atci and Keskin [38] showed that Maxwell model can accurately reproduce the experimental data of ZIF-90/Matrimid and ZIF-90/Ultem MMMs. Erucar and Keskin [39] recently tested a wide variety of theoretical permeation models including Maxwell, modified Maxwell, Bruggeman, Lewis-Nielson, Pal, Felske and modified Felske by comparing their predictions with the available experimental data for IRMOF1/Matrimid and CuBTC/Matrimid MMMs of $\mathrm{CO}_{2} / \mathrm{CH}_{4}$ separations and identified the best predicting theoretical model.

In this work, we used recently developed methods that combine detailed atomistic simulations and theoretical permeation models to make predictions for $\mathrm{H}_{2}$ selectivity and $\mathrm{H}_{2}$ permeability of one hundred and nineteen new MOF-based MMMs composed of seventeen different MOFs and seven different polymers. Our method was validated by comparing the theoretical and experimental results of four different MOF-based MMMs. We identified several promising MOF/polymer combinations which offer very high $\mathrm{H}_{2}$ selectivity and permeability relative to pure polymeric membranes. Based on our results, we discussed how one can estimate the performance of MOF-based MMMs by only examining the gas permeability characteristics of 
pure MOFs. This type of computational screening will be very helpful in identifying the best $\mathrm{MOF} /$ polymer combinations for a specific gas separation.

\section{Details of computational methods:}

\subsection{MOFs and polymers}

We studied seventeen different MOFs in this work. Sixteen of MOFs were chosen from Cambridge Structural Database (CSD) and they are designated by their reference codes (REFCOD) in CSD. These are BACMOH10, BAHGUN, BIMDIL, FOHQUO, GITTIN, LUNBAX, LUNBEB, LUMZUO, MABJOP, MABJUV01, MIHHOA, MIHHIU, MIHHUG, OFERUN, QAMXIL and ZUQPOQ. These materials were selected to represent a wide variety of MOFs showing different combinations of $\mathrm{H}_{2}$ permeabilities and selectivities due to their various structures. We also included a MOF that we recently identified as a very selective material due to its pore structure, MMIF. In our previous studies, we showed that MMIF exhibits very high selectivity for $\mathrm{CO}_{2} / \mathrm{CH}_{4}$ separations as a pure MOF membrane and as filler particles in MMMs [39,40]. More detailed information on structural properties of the MOFs considered in this work is given in Table S1 of Supplementary Data. The polymers we studied in this work lie along Robeson's current upper bound [2] for $\mathrm{H}_{2} / \mathrm{CH}_{4}$ separations: sulfonated polyimide (DAPHFDS(H)), polyimide (6FDA-mMPD), polymide (6FDA-DDBT), Hyflon, Teflon (AF-2400), poly(trimethylsilypropyne-co-phenylpropyne) and polytrimethylsilypropyne. Among these polymers, the last two represent polymers which have almost no $\mathrm{H}_{2}$ selectivity (0.953 and 1.13, respectively) but high $\mathrm{H}_{2}$ permeability (20400 and 17000 Barrer, respectively) whereas sulfonated polyimide represents a polymer having high $\mathrm{H}_{2}$ selectivity (325) but very low permeability (52 Barrer). Others are a wide range of polymers that have moderate $\mathrm{H}_{2}$ selectivity and permeability (selectivity in the range of 5.5121 , permeability in the range of 106-3300 Barrer) for which incorporating a MOF can yield large performance enhancements.

\subsection{Theoretical permeation models}

Several permeation models such as Maxwell, modified Maxwell, Bruggeman, Lewis-Nielson, Pal, Felske and modified Felske are present in the literature to predict gas permeation in MMMs. In our recent study [39], by comparing with many experimental studies, we showed that Maxwell model (modified Felske model) is the best predicting permeation model among the ones considering ideal morphology (non-ideal morphology). Therefore, we used these two models in this study. Maxwell model [41] does not consider packing limit of particles, the effect of particle size distribution, particle shape and aggregation of particles. This model 
predicts gas permeability $(\mathrm{P})$ in a $\mathrm{MOF} /$ polymer membrane based on the volume fraction of MOF particles $(\phi)$, and the permeability ratio $\left(\mathrm{P}_{\mathrm{d}} / \mathrm{P}_{\mathrm{m}}=\lambda_{\mathrm{dm}}\right)$ where $\mathrm{P}_{\mathrm{d}}$ is the permeability of dispersed phase, $\mathrm{P}_{\mathrm{m}}$ is the permeability of continuous phase, $\mathrm{P}_{\mathrm{r}}$ is the relative permeability:

$$
\mathrm{P}_{\mathrm{r}}=\frac{\mathrm{P}}{\mathrm{P}_{\mathrm{m}}}=\left[\frac{2(1-\phi)+(1+2 \phi) \lambda_{\mathrm{dm}}}{(2+\phi)+(1-\phi) \lambda_{\mathrm{dm}}}\right]
$$

Modified Felske model [42] was developed to demonstrate permeation behavior of gases in MMMs by considering the morphology and packing factor of particles:

$$
\begin{gathered}
\mathrm{P}_{\mathrm{r}}=\frac{\mathrm{P}}{\mathrm{P}_{\mathrm{m}}}=\left[\frac{1+2((\beta-\gamma) /(\beta+2 \gamma)) \phi}{1-((\beta-\gamma) /(\beta+2 \gamma)) \phi \varphi}\right] \\
\varphi=1+\left[\frac{\left(1-\phi_{\mathrm{m}}\right)}{\left(\phi_{\mathrm{m}}\right)^{2}}\right] \phi \\
\beta=\left(2+\delta^{3}\right) \lambda_{\mathrm{dm}}-2\left(1-\delta^{3}\right) \lambda_{\mathrm{Im}} \\
\gamma=\left(1+2 \delta^{3}\right)-\left(1-\delta^{3}\right) \lambda_{\mathrm{dI}}
\end{gathered}
$$

This model is based on the two-phase description, the polymer matrix is one phase, the dispersed particles-interface is the other phase ('pseudo-insert' phase) [43]. Here, $\phi_{\mathrm{m}}$ is the maximum packing volume fraction of filler particles and assumed to be 0.64 for random close packing of uniform spheres, $[44,45,46] \delta$ is the ratio of outer radius of interfacial shell to core radius. Shimekit et al. [46] used $\delta$ as 1.18 whereas we used it as 1.11 in this work to get a better agreement with the available experimental data. The percentage average absolute relative error for $\mathrm{CH}_{4}$ permeability in $\mathrm{Cu}$-BPY-HFS/Matrimid membrane was $29 \%$ and $18 \%$ when $\delta$ was equal to 1.18 and 1.11 , respectively. In equation (4), $\lambda_{\mathrm{Im}}$ is the permeability ratio of $\mathrm{P}_{\mathrm{I}} / \mathrm{P}_{\mathrm{m}}, \lambda_{\mathrm{dI}}$ is the permeability ratio of $\mathrm{P}_{\mathrm{d}} / \mathrm{P}_{\mathrm{I}}, \mathrm{P}_{\mathrm{I}}$ is the permeability of the rigidified interphase layer $\left(\mathrm{P}_{\mathrm{m}} / * \beta\right)$. Here, $* \beta$ is the matrix rigidification factor and taken as 3 for gas penetrates in semi-crystalline polymers as reported by Shimekit et al. [46] Modified Felske model turns into Maxwell model when $\phi_{\mathrm{m}}$ and $\delta$ are both equal to 1 .

Both Maxwell model and modified Felske model require permeation of gases through the continuous phase (the polymer matrix) and through the dispersed phase (the filler particles, MOFs). A large set of experimental data is available for gas selectivity and gas permeability of polymers [1,2]. Similar experimental data is not available for MOFs and a key ingredient of 
our results is that gas permeabilities through MOFs were predicted from detailed atomistic simulations as explained in the following section. The $\mathrm{H}_{2}$ selectivity of the MMMs was simply calculated as the ratio of permeabilities of two gas components, $\mathrm{S}_{\mathrm{H} 2 / \mathrm{CH} 4}=\mathrm{P}_{\mathrm{H} 2} / \mathrm{P}_{\mathrm{CH} 4}$.

\subsection{Molecular simulations}

Atomically detailed simulations were used to predict gas permeance through MOFs. Atomic positions of the MOFs were obtained from the experimentally reported crystal files in CSD and rigid MOF frameworks were used. We used universal force field (UFF) [47] for the framework atoms. Spherical Lennard-Jones (LJ) 12-6 potentials were used to model $\mathrm{CH}_{4}$ and $\mathrm{H}_{2}$ [48,49]. Single component adsorption isotherms of each species in MOFs as a function of gas fugacity were computed using Grand Canonical Monte Carlo (GCMC) simulations. Equilibrium molecular dynamics (EMD) simulations at the NVT ensemble (NVT=constant number of molecules, constant volume and constant temperature) were used to compute the loading dependent corrected diffusivities of the same species. The transport diffusivity $\left(D_{t}\right)$ is then defined without any approximation in terms of the corrected diffusivity $\left(\mathrm{D}_{\mathrm{o}}\right)$ and the thermodynamic correction factor [50], where the latter is a partial derivative relating the adsorbate concentration, $c$, and bulk phase fugacity, $f$.

$$
D_{t}(c)=D_{o}(c) \cdot \frac{\partial \ln f}{\partial \ln c}
$$

The thermodynamic correction factor is fully defined once the single component adsorption isotherm of each species is known from GCMC simulations. After GCMC and EMD simulations, steady state fluxes $(\mathrm{J})$ of each gas species through a MOF were calculated using shell description of the membrane based on Fick's law:

$$
\mathrm{J}=-\mathrm{D}_{\mathrm{t}}(\mathrm{c}) \cdot \nabla \mathrm{c}
$$

In this formulation, $\nabla \mathrm{c}$ is the concentration gradient of the adsorbed species based on the difference between the feed and permeate side pressures of the membrane, $\nabla \mathrm{c}=\left(\mathrm{c}^{\text {permeate }}-\mathrm{c}^{\text {feed }}\right) / \mathrm{L} \quad[50,51]$. The gas flux in MOFs is then converted to permeability, $\mathrm{P}$, using pressure drop $(\Delta \mathrm{p})$, and membrane thickness, L, by [52]

$$
\mathrm{P}=\frac{\mathrm{J}}{\Delta \mathrm{p} / \mathrm{L}}
$$


We performed GCMC and EMD simulations for all MOFs at $25^{\circ} \mathrm{C}$ and at a feed (permeate) pressure of 2 bar (vacuum) to be consistent with the experimental data of the pure polymer membranes and MOF-based MMMs. The experimental conditions for the gas permeance measurements of CuBTC-based MMMs synthesized by Car et al. [24] were not specified, therefore we used the same temperature and pressures reported above in modeling of these membranes. The details of GCMC and EMD simulations for predicting permeabilities through MOFs have been described in our earlier studies [39,53].

\section{Results and discussion:}

\subsection{Comparing theoretical results with experimental data}

Figure 1 compares the predictions of Maxwell and modified Felske models with the available experimental data for pure gas permeabilities of $\mathrm{H}_{2}$ and $\mathrm{CH}_{4}$ in four different MMMs: IRMOF-1/Matrimid [26], Cu-BPY-HFS/Matrimid [24], CuBTC/PSF [22], and CuBTC/PDMS [22]. The closed (open) symbols represent predictions of Maxwell (modified Felske) model. The loadings of the MOFs in the MMMs vary from 5\% to 40\%. Figure 1 shows that there is a good agreement between experimental measurements and our theoretical predictions for all MMMs. The modified Felske model makes better predictions than the Maxwell model especially at higher loadings of the filler particles. This is expected because Maxwell model is known to give less accurate results once the volume fraction of the filler particles exceeds 0.2 [54]. The modified Felske model considers non-ideal morphology while Maxwell model assumes ideal morphology based on perfect contact assumption between the polymer and dispersed phase (MOFs). Recent reviews discussed that unexpected voids and defects can cause formation of interfacial phase in the MMMs, therefore using modified Felske model can be more reasonable for the assessment of a MMM's performance [55]. The good agreement between predictions of theoretical models and experimental data indicates that these models are appropriate to estimate the performances of new MOF-based MMMs prior to their fabrication for identifying promising MMM candidates for $\mathrm{H}_{2} / \mathrm{CH}_{4}$ separation.

\subsection{Predicting performances of new MOF-based MMMs}

Figure 2 shows $\mathrm{H}_{2}$ selectivity and $\mathrm{H}_{2}$ permeability of pure polymers and pure MOFs considered in this work. Selectivity and permeability values for each MOF were calculated using detailed atomic simulations as described in Section 2.3 whereas values for polymers were taken from Robeson's large experimental data collection [2]. The line in Figure 2 shows the present upper bound established for polymeric membranes in $\mathrm{H}_{2} / \mathrm{CH}_{4}$ separations. In order to understand the effect of a MOF on the performance of a MMM, we categorized seventeen 
MOFs that we considered in this study into 5 different groups. The first group includes BAHGUN, BIMDIL, MIHHIU, MABJUV01, QAMXIL and ZUQPOQ. The common characteristics of these MOFs are their high $\mathrm{H}_{2}$ permeabilities $\left(5 \times 10^{4}-2.5 \times 10^{5}\right.$ Barrer $)$ and low $\mathrm{H}_{2}$ selectivities (ranging from 1.3 to 21 ). The second group includes materials having high $\mathrm{H}_{2}$ selectivities (33-3186) and high $\mathrm{H}_{2}$ permeabilities (3-5 $\times 10^{4}$ Barrer). The MOFs of this group are BACMOH10, GITTIN, MABJOP and MIHHUG. The third group consists of MOFs showing very high $\mathrm{H}_{2}$ selectivities but low $\mathrm{H}_{2}$ permeabilities $\left(<3 \times 10^{4}\right.$ Barrer $)$. These are FOHQUO, MIHHOA and OFERUN. The fourth group represents MOFs with low $\mathrm{H}_{2}$ permeabilities $\left(<1.8 \times 10^{4}\right.$ Barrer) and low $\mathrm{H}_{2}$ selectivities $(<8)$, LUNBAX, LUNBEB and LUMZUO. We categorized MMIF separately as a material which has an extraordinarily high $\mathrm{H}_{2}$ selectivity and low $\mathrm{H}_{2}$ permeability (981 Barrer). This categorization will be helpful in choosing new MOFs as filler particles because by characterizing a MOF's permeability and selectivity, we will be able to estimate the performance of a MOF-based MMM without detailed calculations as discussed in the following section.

We predicted the performance of new MOF-based MMMs using both Maxwell and modified Felske models. One MOF from each group was chosen as a representative and the results are shown in Figure 3. Results for all other MOF-based MMMs are given in Supplementary Data. Figures 3a,b show that incorporating a MOF such as BAHGUN (from the first group) into polymers increases $\mathrm{H}_{2}$ permeability of polyimides and Hyflon without changing their selectivity significantly. This is expected since this group of MOFs was identified with high permeability and low selectivity. For example, $\mathrm{H}_{2}$ permeability of Hyflon increases from 3300 to 6716 Barrer when the volume fraction of BAHGUN is equal to 0.3. BAHGUN/Hyflon MMM can exceed the upper bound due to permeability enhancement. MOFs of the first group increase both the selectivity and permeability of polytrimethylsilylpropynes (PTMSPs) significantly since these MOFs have higher $\mathrm{H}_{2}$ selectivity and permeability than pure PTMSPs.

Figures 3c,d present the effects of a MOF, MIHHUG, exhibiting high selectivity and high permeability. This type of MOFs has positive impact both on selectivity and permeability of all polymers, but enhancements are more pronounced for Teflon and PTMSPs. For example, the selectivity (the permeability) of Teflon increases from 5.5 (3300 Barrer) to 11 (5233 Barrer) at a 0.2 volume fraction of MIHHUG. Selecting MOFs from the second group for making MOF-based MMMs is a very good strategy because even a small fraction of these MOFs can carry all the polymers well above the upper bound. A comparison between Figures 
3a,b and 3c,d highlights that the characteristics of MOFs are important especially for the polymers lying at the bottom of the upper bound.

Figures 3e,f show that MOFs from the third group with high selectivity and low permeability characteristics such as FOHQUO can increase the selectivity of PTMSPs without making a significant change in their permeability. This result is expected since FOHQUO and other members of the third group have similar permeabilities to those of PTMSPs. One important aspect of this figure is the more optimistic predictions of Maxwell model compared to modified Felske model as discussed in Section 3.1 and in a previous study [46]. For example, Maxwell model predicts that $\mathrm{H}_{2}$ permeability is increased from 20400 to 20800 Barrer whereas modified Felske model predicts that it decreases to 19400 Barrer when FOHQUO at a volume fraction of 0.2 is embedded into polytrimethylyslypropyne-cophenylpropyne (PTMSP-cPP).

MOFs categorized as members of the fourth group represent materials with low selectivity and low permeability compared to the other groups. Figures $3 \mathrm{~g}$, h indicate that incorporation of this type of MOFs into different polymers gives varying results based on the type of the polymer. For example, LUNBEB increases the permeability of polyimides without changing their selectivities significantly, increases both selectivity and permeability of Teflon, increases the selectivities of PTMSPs but decreases their permeability. This result underlines the importance of MOF/polymer matching. Matching LUNBEB with Teflon will be a 'win-win' case, whereas matching it with PTMSPs improve the selectivity at the expense of permeability. One can conclude that members of the fourth group are useful MOFs as filler particles for polymeric membranes which already have high selectivity but require higher permeability to exceed the upper bound such as polyimides. Finally, we examined the impact of a MOF which has an extraordinarily high $\mathrm{H}_{2}$ selectivity, MMIF. As expected, even small volume fractions of MMIF can significantly enhance both selectivity and permeability of polyimides. Figures 3i,j show that selectivity (permeability) of polyimide 6FDA-DDBT increases from 78.8 (156 Barrer) to 156 (225 Barrer) at a MOF volume fraction of 0.2. When MMIF was added into Teflon and PTMSPs, selectivity increase is obtained at the expense of permeability since MMIF has lower permeability than these polymers. For example, permeability of Teflon (PTMSPs) decreases from 3300 (23200) to 2467 (14616) Barrer although selectivity is increased from 5.5 (0.99) to 6.8 (1.03). Matching highly selective MOFs with different types of polyimides will result in membranes with very promising properties for $\mathrm{H}_{2}$ separation. 
So far we examined the impact of MOFs as filler particles in the polymers which establish the upper bound for $\mathrm{H}_{2} / \mathrm{CH}_{4}$ separations. Since these polymers were close to the upper bound, it was relatively easy to carry them above the upper bound by adding a small volume fraction of MOFs. In the next section, we considered the most widely used polymers Matrimid, PSF and PDMS, which are away from the upper bound, and described the required characteristics of MOFs to enhance the separation performances of these three polymers.

\subsection{Selecting appropriate MOF/polymer pairs for high performance MMMs}

In order to select appropriate MOF/polymer pairs to achieve high performance MMMs, we investigated real polymer/hypothetical MOF systems and hypothetical polymer/real MOF systems. Figure 4 shows the performances of MMMs composed of hypothetical MOFs and three commercial polymers, Matrimid, PSF and PDMS. We specifically focused on these polymers because most of the fabricated MOF-based MMMs employed these polymers as reported in the introduction part. The Matrimid, PSF and PDMS can be thought as examples of polymers having high selectivity/low permeability (83.3, 17.5 Barrer), medium selectivity/medium permeability (24.5/9.7 Barrer), and low selectivity/high permeability (0.74/577.6 Barrer), respectively [22,26]. Three different scenarios were considered to improve the performance of polymeric membranes by incorporating MOFs: 1)enhancing selectivity without changing permeability 2)improving both selectivity and permeability 3)increasing permeability without changing selectivity. The selectivity and permeability properties of the hypothetical MOFs to achieve these scenarios were determined by using Maxwell and modified Felske models and these hypothetical MOFs were shown in Figure 4 as MOF-1 (scenario 1), MOF-2 (scenario 2), MOF-3 (scenario 2) and MOF-4 (scenario 3). We also represented the location of the real MOF groups in Figure 4. The open symbols represent performances of MMMs having hypothetical MOFs as filler particles and the close symbols are the performances of pure polymers and pure MOFs. The volume fraction of MOFs was limited to 0.5 to be consistent with the experiments.

Figure 4a,b shows that pairing Matrimid with MOF-1 (MOF-4) increases only the selectivity (permeability) and helps the MMM to approach the upper bound without exceeding it. On the other hand, it is possible to carry Matrimid above the upper bound by using a MOF which has similar properties to MOF-2. In fact, MMIF (shown as 5 in the figure) has a higher selectivity than MOF-2, therefore matching Matrimid with MMIF would result in a high performance MMM. The MOFs of the third group can also carry Matrimid above the upper bound since they have higher selectivity and permeability than MOF-3. Figure 4c-f presents that choosing 
a MOF from the third group can increase both selectivity and permeability of PSF and PDMS membranes since hypothetical MOF-3 falls into the third group. Using MOFs from the first or fourth group as filler particles in PSF and PDMS enhances $\mathrm{H}_{2}$ permeability of both polymers. In contrast to Matrimid, none of the hypothetical MOFs shown in Figure 4c,d can carry PSF above the upper bound. This is due to the lower selectivity and permeability characteristics of PSF compared to Matrimid. Finally, selecting MOFs from the first and fourth groups will be also useful for PDMS because these MOFs will enhance both $\mathrm{H}_{2}$ selectivity and $\mathrm{H}_{2}$ permeability of pure polymer. As we discussed before, Maxwell model predicts higher gas permeability compared to modified Felske model in Figure 4. The main conclusion of this figure is that various MOFs exist to improve $\mathrm{H}_{2}$ selectivity and/or permeability of commercial polymers and one can estimate how a MOF-based MMM would behave by identifying the location of the MOF in Figure 4.

After studying widely used commercial polymers, we examined hypothetical polymers to understand which polymers can reap the largest advantages when used in combination with MOFs. In Figure 5, we considered hypothetical polymers that lie along the Robeson's upper bound for $\mathrm{H}_{2} / \mathrm{CH}_{4}$ separation. By specifying the position of a polymer along the line we defined the information required to predict MMMs' performances. For each hypothetical polymer, we used MIHHOA and ZUQPOQ as filler particles to represent a highly selective and a weakly selective MOF, respectively. The open (closed) stars represent the modified Felske model predictions for the performance of ZUQPOQ/polymer (MIHHOA/polymer) MMMs. Figure 5 shows that if the polymer has a high selectivity for $\mathrm{H}_{2}$ but low permeability (polymer 1), adding a MOF can enhance the polymeric membrane's permeability with little or no change in the membrane's selectivity. In this limit, the identity of the MOF appears to be unimportant. Similarly, the identity of the MOF is not important if the polymer matrix has a very low selectivity but high permeability such as polymer 6. In this case, both MOFs will increase its selectivity at the expense of permeability. For polymers having medium selectivity and permeability (such as polymers 2, 3), the identity of MOF is essential. For example, MIHHOA increases both permeability and selectivity whereas ZUQPOQ can only improve permeability without any important change in selectivity. The extreme example is a polymer that is very permeable but has a very low selectivity like polymer 5. For this polymer, the identity of the MOF used in a MMM plays a critical role. A permeable but unselective MOF, such as ZUQPOQ, increases permeability but decreases selectivity slightly. On the 
other hand, a highly selective MOF like MIHHOA increases selectivity but slightly decreases the permeability.

\section{Conclusions:}

In this study, we applied recently developed methods that combine detailed atomic simulations and theoretical permeation models to make predictions for the properties of MOFbased $M M M s$ in $\mathrm{H}_{2} / \mathrm{CH}_{4}$ separations. We first showed that this approach gives good agreement with the experimental results of four fabricated MOF-based MMMs and then used this approach to estimate the performances of one hundred and nineteen new MOF-based MMMs. It is important to describe the limitations of our approach. One effect that our approach did not account for is that a good fraction of the MOF materials that we considered in this work do not have three dimensional porosities. These materials have non-isotropic transport properties. Kang et al.[56] recently examined one dimensional molecular transport in tubular filler particles and presented an analytical model to estimate permeation in composite membranes with tubular fillers. They showed that the sensitivity of the permeability enhancement to the filler orientation becomes higher when the permeability of the filler is somewhat similar to that of the polymer. The MOF fillers we considered in this work has generally higher permeabilities than the polymers, therefore we expect that permeability enhancement does not strongly depend on the orientation of MOFs in composite membranes. Kang's model is especially useful to include the effects of defects and pinholes that sometimes form under non-ideal membrane fabrication conditions. Our calculations do not make any predictions about the long-term stability of MOF-based MMMs. The stability of these membranes is a serious issue in practical applications and it is likely that this issue is more straightforward to address experimentally. The encouraging point is that early experimental studies for fabricated MOF-based MMMs reported that these membranes are stable and there is a good adhesion between MOF particles and polymers.

Our earlier work on MMMs illustrated that a MOF/polymer combination with excellent separation properties for one gas separation might have less useful properties for other gas separations [37]. The same conclusion was found to be valid in this work. For example, in a recent study [39], we showed that BAHGUN is a very promising material due to its high $\mathrm{CO}_{2}$ selectivity $\left(>10^{4}\right)$ for separation of $\mathrm{CO}_{2}$ from $\mathrm{CH}_{4}$. However, in this work we found that its $\mathrm{H}_{2}$ selectivity from $\mathrm{CH}_{4}$ is not very high, 10. By comparing the results of our previous study and this study, we can conclude that materials having large pores connected with narrow pore windows such as MMIF are the highly promising candidates both for separation of $\mathrm{CO}_{2}$ from 
$\mathrm{CH}_{4}$ and for separation of $\mathrm{H}_{2}$ from $\mathrm{CH}_{4}$. This is due to the very slow diffusion of $\mathrm{CH}_{4}$ molecules in the narrow pores which are similar in size to the kinetic diameter of $\mathrm{CH}_{4}$ molecule [40].

In summary, our results showed that there is a wide range of polymers that have moderate selectivity and moderate permeability for which adding the appropriate MOF can yield large performance enhancements. The approach we introduced here will be useful for understanding which MOFs from the huge numbers of available materials would be worthwhile targets for experimental efforts in developing new MOF-based MMMs.

\section{Nomenclature:}

$\begin{array}{ll}c & \text { adsorbate concentration } \\ D_{t} & \text { transport diffusivity } \\ D_{o} & \text { corrected diffusivity } \\ f & \text { bulk phase fugacity } \\ \text { J } & \text { steady state flux } \\ \text { L } & \text { membrane thickness } \\ \text { MMM } & \text { mixed matrix membrane } \\ \text { MOF } & \text { metal organic framework } \\ P & \text { gas permeability in a MOF/polymer membrane } \\ P_{d} & \text { gas permeability of dispersed phase } \\ \mathrm{P}_{\mathrm{I}} & \text { gas permeability of the rigidified interphase layer } \\ \mathrm{P}_{\mathrm{m}} & \text { gas permeability of continuous phase } \\ \mathrm{P}_{\mathrm{r}} & \text { relative gas permeability } \\ \mathrm{S} & \text { selectivity }\end{array}$

Greek letters

$\nabla \mathrm{c} \quad$ concentration gradient of the adsorbed species

$\Delta \mathrm{p} \quad$ pressure drop

$* \beta \quad$ matrix rigidification factor

$\lambda_{\mathrm{dm}} \quad$ permeability ratio of $\mathrm{P}_{\mathrm{d}} / \mathrm{P}_{\mathrm{m}}$

$\lambda_{\text {Im }} \quad$ permeability ratio of $\mathrm{P}_{\mathrm{I}} / \mathrm{P}_{\mathrm{m}}$

$\lambda_{\mathrm{dI}} \quad$ permeability ratio of $\mathrm{P}_{\mathrm{d}} / \mathrm{P}_{\mathrm{I}}$

$\phi \quad$ volume fraction of filler particles 
$\phi_{\mathrm{m}} \quad$ maximum packing volume fraction of filler particles

$\delta \quad$ ratio of outer radius of interfacial shell to core radius

$\varphi \quad$ a parameter described for volume fraction of filler particles

$\beta \quad$ a parameter for ratio of the interphase permeability to the polymer permeability

$\gamma \quad$ a parameter described for ratio of the interphase thickness to the particle radius

Acknowledgment: Financial support provided by the European Commission Marie Curie International Re-integration Grant FP7-PEOPLE-2010-RG (COMMOF-268142) is gratefully acknowledged.

Supplementary data: Supplementary data associated with this article is available.

\section{References:}

1. L.M. Robeson, Correlation of separation factor versus permeability for polymeric membranes. J. Membr. Sci. 62 (1991) 165-185.

2. L.M. Robeson, The upper bound revisited. J. Membr. Sci. 320 (2008) 390-400.

3. B.D. Freeman, Basis of permeability/selectivity tradeoff relations in polymeric gas separation membranes. Macromolecules 32 (1999) 375-380.

4. L.M. Robeson, B.D. Freeman, D.R. Paul, B.W. Rowe, An empirical correlation of gas permeability and permselectivity in polymers and its theoretical basis. J. Membr. Sci. 341 (2009) 178-185.

5. M.A. Snyder, M. Tsapatsis, Hierarchical nanomanufacturing: From shaped zeolite nanoparitcles to high-performance separation membranes. Angew. Chem. Int. Ed. 46 (2007) $7560-7573$.

6. B.J. Hinds, N. Chopra, T. Rantell, R. Andrews, V. Gavalas, L. Bachas, Aligned multiwalled carbon nanotube membranes. Science 303 (2004) 62-65.

7. A.I. Skoulidas, D.M. Ackerman, J.K. Johnson, D.S. Sholl, Rapid transport of gases in carbon nanotubes. Phys. Rev. Lett. 89 (2002) 185901-185904.

8. A. Julbe, Zeolite membranes synthesis, characterization and application, Introduction to zeolite science and practice, in: H.B. J. Cejka, A. Corma, F. Schuth (Eds.) Elsevier 168 (2007) 181-219.

9. C.M. Zimmerman, A. Singh, W.J. Koros, Tailoring mixed matrix composite membranes for gas separations. J. Membr. Sci. 137 (1997) 145-154.

10. R. Mahajan, W.J. Koros, Factors controlling successful formation of mixed-matrix gas separation materials. Ind. Eng. Chem. Res. 39 (2000) 2692-2696.

11. T.C. Merkel, B.D. Freeman, R.J. Spontak, Z. He, I. Pinnau, P. Meakin, A.J. Hill, Ultrapermeable, reverse-selective nanocomposite membranes. Science 296 (2002) 519-521.

12. D.Q. Vu, W.J. Koros, S.J. Miller, Mixed matrix membranes using carbon molecular sieves. I. Preparation and experimental results. J. Membr. Sci. 211 (2003) 311-334.

13. M. Das, J.D. Perry, W.J. Koros, Gas-transport-property performance of hybrid carbon molecular sieve-polymer materials. Ind. Eng. Chem. Res. 49 (2010) 9310-9321.

14. T.T. Moore, W.J. Koros, Sorption in zeolites modified for use in organic-inorganic hybrid membranes. Ind. Eng. Chem. Res. 47 (2008) 591-598. 
15. H.J.C. Hennepe, W.B.F. Boswerger, D. Bargeman, M.H.V. Mulder, C.A. Smolders, Zeolite-filled silicon rubber membranes - experimental determination of concentration profiles. J. Membr. Sci. 89 (1994) 185-196.

16. E. Okumus, T. Gurkan, L. Yilmaz, Development of a mixed matrix membrane for pervaporation. Sep. Sci. Technol. 29 (1994) 2451-2473.

17. I.F.J. Vankelecom, C. Dotremont, M. Morobe, J.B. Uytterhoeven, C. Vandecasteele, Zeolite-filled pdms membranes. 1. Sorption of halogenated hydrocarbons. J. Phys. Chem. B 101 (1997) 2154-2159.

18. T. Chung, S. Chan, R. Wang, Z. Lu, C. He, Characterization of permeability and sorption in matrimid/c60 mixed matrix membranes. J. Membr. Sci. 211 (2003) 91-99.

19. S. Qiu, L. Wu, G. Shi, L. Zhang, H. Chen, C. Gao, Preparation and pervaporation property of chitosan membrane with functionalized multiwalled carbon nanotubes. Ind. Eng. Chem. Res. 49 (2010) 11667-11675.

20. M. Eddaoudi, H. Li, O.M. Yaghi, Highly porous and stable metal-organic frameworks: Structure design and sorption properties. J. Am. Chem. Soc. 122 (2000) 1391-1397.

21. R. Banerjee, H. Furukawa, D. Britt, C. Knobler, M. O'Keeffe, O.M. Yaghi, Control of pore size and functionality in isoreticular zeolitic imidazolate frameworks and their carbon dioxide selective capture properties. J. Am. Chem. Soc. 131 (2009) 3875-3877.

22. A. Car, C. Stropnik, K.V. Peinemann, Hybrid membrane materials with different metal-organic frameworks (MOFs) for gas separation. Desalination 200 (2006) 424426.

23. J. Hu, H. Cai, H. Ren, Y. Wei, Z. Xu, H. Liu, Y. Hu, Mixed-matrix membrane hollow fibers of $\mathrm{cu}_{3}(\mathrm{btc})_{2} \mathrm{MOF}$ and polyimide for gas separation and adsorption. Ind. Eng. Chem. Res. 49 (2010) 12605-12612.

24. Y. Zhang, I.H. Musselman, J.P. Ferraris, K.J. Balkus, Gas permeability properties of matrimid ${ }^{\circledR}$ membranes containing the metal-organic framework $\mathrm{Cu}-\mathrm{BPY}-\mathrm{HFS}$. J. Membr. Sci. 313 (2008) 170-181.

25. S. Basu, A. Cano-Odena, I.F.J. Vankelecom, Asymmetric Matrimid/[Cu $\left.\mathrm{Cu}_{3}(\mathrm{btc})_{2}\right]$ mixed matrix membranes for gas separations. J. Memb. Sci. 362 (2010) 478-487.

26. E.V. Perez, K.J. Balkus, J.P. Ferraris, I.H. Musselman, Mixed-matrix membranes containing MOF-5 for gas separations. J. Membr. Sci. 328 (2009) 165-173.

27. R. Adams, C. Carson, J. Ward, R. Tannenbaum, W. Koros, Metal organic framework mixed matrix membranes for gas separations. Micro. Meso. Mater. 131 (2010) 13-20.

28. T.X. Yang, Y.C. Xiao, T.S. Chung, Poly-/metal-benzimidazole nano-composite membranes for hydrogen purification. Energy Environ. Sci. 4 (2011) 4171-4180.

29. K. Diaz, L. Garrido, M. Lopez-Gonzalez, L.F. del Castillo, E. Riande, $\mathrm{CO}_{2}$ transport in polysulfone membranes containing zeolitic imidazolate frameworks as determined by permeation and PFG NMR techniques. Macromolecules 43 (2010) 316-325.

30. X.L. Liu, Y.S. Li, G.Q. Zhu, Y.J. Ban, L.Y. Xu, W.S. Yang, An organophilic pervaporation membrane derived from metal-organic framework nanoparticles for efficient recovery of bio-alcohols. Angew. Chem. Int. Edit. 50 (2011) 10636-10639.

31. C. Zhang, Y. Dai, J.R. Johnson, O. Karvan, W.J. Koros, High performance ZIF8/6FDA-DAM mixed matrix membrane for propylene/propane separations. J. Membr. Sci. 389 (2012) 34-42.

32. T.-H. Bae, J.S. Lee, W. Qiu, W.J. Koros, C.W. Jones, S. Nair, A high-performance gas-separation membrane containing submicrometer-sized metal-organic framework crystals. Angew. Chem. Int. Ed. 49 (2010) 9863-9866.

33. S. Basu, M. Maes, A.Cano-Odena, L. Alaerts, D.E.D. Vos, I.F.J. Vankelecom, Solvent resistant nanofiltration (SRNF) membranes based on metal-organic frameworks. J. Memb. Sci. 344 (2009) 190-198. 
34. S. Basu, A. Cano-Odena, I.F.J. Vankelecom, MOF-containing mixed-matrix membranes for $\mathrm{CO}_{2} / \mathrm{CH}_{4}$ and $\mathrm{CO}_{2} / \mathrm{N}_{2}$ binary gas mixture separations. Sep. Purif. Technol. 81 (2011) 31-40.

35. Y. Liu, Z. Ng, E.A. Khan, H.-K. Jeong, C.-B. Ching, Z. Lai, Synthesis of continous MOF-5 membranes on porous alpha-alumina substrates. Micropor. Mesopor. Mater. 118 (2009) 296-301.

36. S. Keskin, D.S. Sholl, Assessment of a metal-organic framework membrane for gas separations using atomically detailed calculations: $\mathrm{CO}_{2}, \mathrm{CH}_{4}, \mathrm{~N}_{2}, \mathrm{H}_{2}$ mixtures in MOF-5. Ind. Eng. Chem. Res. 48 (2009) 914-922.

37. S. Keskin, D.S. Sholl, Selecting metal organic frameworks as enabling materials in mixed matrix membranes for high efficiency natural gas purification. Energy Environ. Sci. 3 (2010) 343-351.

38. E. Atci, S. Keskin, Atomically detailed models for transport of gas mixtures in ZIF membranes and ZIF/polymer composite membranes. Ind. Eng. Chem. Res. DOI: 10.1021/ie202530f (2012).

39. I. Erucar, S. Keskin, Screening metal organic framework-based mixed matrix membranes for $\mathrm{CO}_{2} / \mathrm{CH}_{4}$ separations. Ind. Eng. Chem. Res. 50 (2011) 12606-12616.

40. S. Keskin, High $\mathrm{CO}_{2}$ selectivity of a microporous metal-imidazolate framework: A molecular simulation study. Ind. Eng. Chem. Res. 50 (2011) 8230-8236.

41. J.C. Maxwell, A treatise on electricity and magnetism. Dover Publications. New York, 1954.

42. J.D. Felske, Effective thermal conductivity of composite spheres in a continuous medium with contact resistance. Int. J. Heat Mass Transfer. 47 (2004) 3453-3461.

43. T.T. Moore, R. Mahajan, D.Q. Vu, W.J. Koros, Hybrid membrane materials comprising organic polymers with rigid dispersed phases. AIChE J. 50 (2004) 311321.

44. R. Pal, Permeation models for mixed matrix membranes. J. Colloid Interface Sci. 317 (2008) 191-198.

45. M.A. Aroon, A.F. Ismail, T. Matsuura, M.M. Montazer-Rahmati, Performance studies of mixed matrix membranes for gas separation: A review. Sep. Purify. Tech. 75 (2010) 229-242.

46. B. Shimekit, H. Mukhtara, T. Murugesan, Prediction of the relative permeability of gases in mixed matrix membranes. J. Memb. Sci. 373 (2011) 152-159.

47. A.K. Rappe, C.J. Casewit, K.S. Colwell, W.A. Goddard, W.M. Skiff, UFF, a full periodic table force field for molecular mechanics and molecular dynamics simulations. J. Am. Chem. Soc. 114 (1992) 10024-10035.

48. M.G. Martin, J.I. Siepmann, Predicting multicomponent phase equilibria and free energies of transfer for alkanes by molecular simulation. J. Am. Chem. Soc. 119 (1997) 8921- 8924.

49. V. Buch, Path-integral simulations of mixed para-d-2 and ortho-d-2 clusters - the orientational effects. J. Chem. Phys. 100 (1994) 7610-7629.

50. D.S. Sholl, Understanding macroscopic diffusion of adsorbed molecules in crystalline nanoporous materials via atomistic simulations. Acc. Chem. Res. 39 (2006) 403-411.

51. J.A. Wesselingh, R. Krishna, Mass transfer in multicomponent mixtures. Delft University Press. Delft, 2000.

52. R.E. Kesting, A.K. Fritzsche, Polymeric gas separation membranes. John Wiley \& Sons, Inc. New York, 1993.

53. S. Keskin, D.S. Sholl, Screening metal-organic framework materials for membranebased methane/carbon dioxide separations. J. Phys. Chem. C 111 (2007) 14055-14059. 
54. D.Q. Vu, W.J. Koros, S.J. Miller, Mixed matrix membranes using carbon molecular sieves. II. Modeling permeation behavior. J. Membr. Sci. 211 (2003) 335-348.

55. S. Basu, A.L. Khan, A.Cano-Odena, C. Liub, I.F.J. Vankelecom, Membrane-based technologies for biogas separations. Chem. Soc. Rev. 39 (2010) 750-768.

56. D.-Y. Kang, C.W. Jones, S. Nair, Modeling molecular transport in composite membranes with tubular fillers. J. Membr. Sci. 381 (2011) 50-63.

Figure 1. Comparisons between experimental measurements and theoretical predictions for permeabilities of a) $\mathrm{H}_{2}$ and b) $\mathrm{CH}_{4}$ in IRMOF-1/Matrimid, CuBTC/PSF, CuBTC/PDMS and $\mathrm{Cu}-\mathrm{BPY}-\mathrm{HFS} / \mathrm{Matrimid}$ MMMs. Experimental data is taken from references [22,24,26].

Figure 2. $\mathrm{H}_{2}$ selectivity and $\mathrm{H}_{2}$ permeability of pure polymers and pure MOFs considered in this work.

Figure 3. Predictions of Maxwell and modified Felske models for $\mathrm{H}_{2}$ selectivity and $\mathrm{H}_{2}$ permeability of MOF-based MMMs. a-b)BAHGUN c-d)MIHHUG e-f)FOHQUP gh)LUNBEB i-j)MMIF. Filled symbols represent performances of pure polymers and pure MOFs, stars represent performances of MMMs with filler volume fractions of $0.1,0.2,0.3$, 0.4 and 0.5 .

Figure 4. Effects of hypothetical MOFs on the performance of a-b)Matrimid, c-d)PSF and ef)PDMS-based MMMs. The closed symbols represent pure polymers and pure MOFs. The open symbols are the predictions of theoretical models for the performances of MMMs with filler volume fractions of $0.1,0.2,0.3,0.4$ and 0.5 .

Figure 5. Predictions of modified Felske model for the performances of hypothetical polymer/MOF membranes. The open (closed) stars represent the predictions for the performance of ZUQPOQ/polymer (MIHHOA/polymer) MMMs. 